\title{
Metabolic cost of generating horizontal forces during human running
}

\author{
YOUNG-HUI CHANG AND RODGER KRAM \\ Locomotion Laboratory, Department of Integrative Biology, \\ University of California, Berkeley, California 94720-3140
}

\begin{abstract}
Chang, Young-Hui, and Rodger Kram. Metabolic cost of generating horizontal forces during human running. J . Appl . Physiol. 86(5): 1657-1662, 1999.-Previous studies have suggested that generating vertical force on the ground to support body weight (BWt) is the major determinant of the metabolic cost of running. Because horizontal forces exerted on the ground are often an order of magnitude smaller than vertical forces, some have reasoned that they have negligible cost. U sing applied horizontal forces (AHF ; negative is impeding, positive is aiding) equal to $-6,-3,0,+3,+6,+9,+12$, and $+15 \%$ of $\mathrm{BWt}$, we estimated the cost of generating horizontal forces while subjects were running at $3.3 \mathrm{~m} / \mathrm{s}$. We measured rates of oxygen consumption $\left(\mathrm{VO}_{2}\right)$ for eight subjects. We then used a force-measuring treadmill to measure ground reaction forces from another eight subjects. With an AHF of $-6 \% \mathrm{BWt} \mathrm{VO}_{2}$ increased $30 \%$ compared with normal running, presumably because of the extra work involved. With an AHF of $+15 \%$ BWt, the subjects exerted $\sim 7.0 \%$ less propulsive impulse and exhibited a $33 \%$ reduction in $\mathrm{VO}_{2}$. Our data suggest that generating horizontal propulsive forces constitutes more than one-third of the total metabolic cost of normal running.
\end{abstract}

biomechanics; locomotion; ground reaction forces; energetic cost

IN HUMANS AND OTHER LEGGED animals, the need to generate muscular force to support body weight (BWt) is a major determinant of the metabolic cost of steadyspeed running. Taylor and colleagues (22) found a linear and proportional relationship between vertical loading with added mass and the rate of oxygen consumption $\left(\mathrm{VO}_{2}\right)$ in humans and several other species. Additionally, Farley and McMahon (7) observed a proportional relationship between metabolic cost and the average vertical ground reaction force (GRF) using simulated reduced gravity to "unweight" human subjects. Kram and Taylor (12) showed that, in a variety of species across a wide range of speeds, the metabolic cost of running was proportional to the weight supported. These studies concluded that the vertical GRF (as opposed to the horizontal GRF) is a major determinant of the metabolic cost for steady-speed, level running. This seems reasonable because the peak vertical GRFs for running are an order of magnitude greater than peak horizontal GRFs (17), yet anyone who has run on a windy day intuitively knows that external horizontal forces can substantially affect the metabolic cost of running.

\footnotetext{
The costs of publication of this article were defrayed in part by the payment of page charges. The article must therefore be hereby marked "advertisement" in accordance with 18 U.S.C. Section 1734 solely to indicate this fact.
}

There have been only a few studies on the metabolic significance of horizontal force production during steadyspeed running. Using a wind tunnel to apply horizontal impeding forces, Pugh (19) showed that the metabolic cost of treadmill running increased with the square of head-wind velocity, i.e., approximately proportional to the applied force. Others have found that metabolic cost increases proportionally with an increase in external work performed whilethesubject is running against an impeding force applied via a harness $(4,13,23)$. We are aware of only one study that has investigated the metabolic effects of horizontal aiding forces. Davies (5) compared the metabolic cost of running with wind resistance vs. wind assistance for three subjects. He did not, however, directly measure forces applied to the runner, nor did he measure the forces exerted by the runner. Thus there is little biomechanical information available to explain the metabolic changes involved with horizontal loading.

Our aim was to alter the horizontal forces generated by the runner on the ground and to measure the corresponding changes in metabolic cost. We altered the horizontal forces generated by the runner by using an applied horizontal force (AHF). We compared the rates of $\mathrm{VO}_{2}$ and the integrated horizontal GRFs (impulses) for subjects running as we applied external horizontal aiding forces (+AHF) or external horizontal impeding forces (-AHF) at the waist. In doing so, we measured the changes in the propulsive and braking forces that subjects generated to compensate for the AHF. We then partitioned the relative importance of the horizontal propulsive forces vs. the horizontal braking forces generated during running. By measuring horizontal GRFs, we extended our understanding of running beyond previous studies of vertical and horizontal loading that did not measure the horizontal GRFs.

Our rationale for this study was that, when we provided an external horizontal aiding force, the reduction in $\mathrm{VO}_{2}$ would reflect the metabolic cost of generating the horizontal propulsive GRF during normal running. We anticipated that applying an impeding force would increase the $\mathrm{VO}_{2}$ and that an aiding force would decrease the $\mathrm{VO}_{2}$. Nevertheless, there is a strong correlation between vertical GRF and metabolic cost $(7,22)$, and our experiment did not alter these vertical forces. Thus our null hypothesis was that the absolute cost of generating horizontal forces on the ground would be relatively small and a minor determinant of the metabolic cost of running.

\section{METHODS}

Subjects. Experiments took place in two independent stages: metabolic measurements and biomechanical measurements. 
Subjects gave informed consent before participating in the experiment, and the protocol was approved by the University of California Committee for the Protection of Human Subjects. F or the metabolic experiments, we collected data on five men and three women ranging in age from 21 to $40 \mathrm{yr}$ [ $27 \pm 7$ (SD) yr]. Body mass ranged from 51.5 to $97.0 \mathrm{~kg}$ $(68.6 \pm 15.5 \mathrm{~kg})$. For the biomechanics experiments, we collected data on four men and four women ranging in age from 20 to $36 \mathrm{yr}(25 \pm 5 \mathrm{yr}$ ). Body mass of these subjects ranged from 55.8 to $81.5 \mathrm{~kg}(65.8 \pm 9.3 \mathrm{~kg})$. All subjects were well-trained recreational runners. One year after collecting the $\mathrm{VO}_{2}$ data, we completed development of a force-treadmill capable of measuring vertical and horizontal GRFs (11). Because the subjects from the metabolic stage of the study were no longer available to participate in the biomechanical data collection, we recruited a new pool of subjects. The ideal experimental design would have been to collect both sets of data simultaneously from a single pool of subjects. Nevertheless, our specially designed force-measuring treadmill (11) had not been invented or built by the time the metabolic data were collected. It would have been possible to collect new metabolic data; however, we felt it was unnecessary, because all of the subjects showed the same pattern of response to the AHFs. Therefore, it is unlikely that either our results or our general conclusions would have changed substantially had we again collected the metabolic data.

Protocol. For the metabolic measurements, we habituated subjects to treadmill running within 7 days of actual data collection in accordance with procedures for kinematic accommodation to treadmill running (21). During the experimental sessions, subjects ran at a speed of $3.3 \mathrm{~m} / \mathrm{s}$ on a motorized treadmill (Quinton 18-60) while we applied horizontal forces equal to $-6,-3,+3,+6,+9,+12$, and $+15 \%$ of the subject's BWt. A negative value indicates an impeding AHF and a positive value indicates an aiding AHF. The order of applying aiding and impeding AHFs was randomized for each subject. Each data-acquisition trial lasted $8 \mathrm{~min}$, and $\mathrm{VO}_{2}$ measurements were averaged for the last $4 \mathrm{~min}$. Subjects rested 6 min between trials except when waiting for the treadmill belt direction to be reversed (which typically lasted 10-15 min). Subjects ran with zero AHF at both the start and end of the experiment. Although we did not measure maximal $\mathrm{VO}_{2}$ of the subjects, we determined that the subjects were exercising at a moderate-intensity level (18) because steady state was achieved within $3 \mathrm{~min}$. Additionally, $\mathrm{VO}_{2}$ values for the zero AHF trials at the beginning and end of the experiment differed by $<1 \%$. For the biomechanical measurements, the protocol was similar to the metabolic experiment with the exception that trials lasted only a few minutes, and this second group of subjects ran on a specially designed forcemeasuring treadmill (11). Data were collected after allowing the subjects to run for $1 \mathrm{~min}$ at each condition. Morgan and colleagues (16) have previously shown that human running kinematic data are highly reliable and exhibit little variability over time.

Horizontal pulling apparatus. Weapplied aiding and impeding forces to the subjects via a waist belt worn near the center of mass (Fig. 1). The waist belt was connected in series with spring el ements composed of rubber tubing that were stretched over a series of low-friction pulleys. The rubber tubing was stretched to two to three times its resting length so that small changes in length would not substantially change the applied force. Thus nearly constant horizontal forces were applied to the subjects. The magnitude of these AHFs was adjusted by altering the number of spring elements in parallel and by adjusting the length of the spring element with a hand winch on the other end of the line.

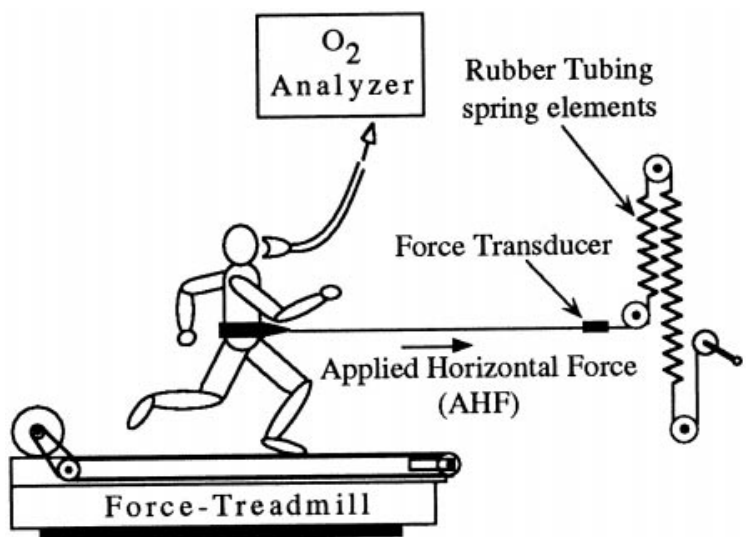

Fig. 1. Schematic of experimental setup. Rubber tubing was stretched over low-friction pulleys with a hand winch to apply a near-constant applied horizontal force (AHF) to subjects running on a treadmill. Aiding AHF is shown. Treadmill belt and running direction were reversed to apply impeding forces. Note that force-treadmill data were collected separately from metabolic data but are shown together here for convenience.

We monitored the AHF with a force transducer in series with the line from the waist belt. Subjects were provided with a digital readout of the AHF and asked to maintain the appropriate level of force while running. AHFs were maintained within $\pm 5 \%$ of the desired value across the entire range of applied-force conditions. Our method of applying horizontal forces differed from previous methods that used a rope connecting the subject to various weights hung over a low-friction pulley $(4,13,23)$. The fore-aft oscillations of the center of mass while the subject is running on a treadmill could create substantial fluctuations in the AHF when an inertial mass is used to apply the force. Our design had a very-low-inertia compliant spring that resulted in a nearly constant AHF.

We calculated the average vertical GRF per step as well as the average peak vertical GRF per step for each subject to verify that only horizontal GRF s were altered by our apparatus. Across all conditions of AHF, neither the average vertical GRF nor the average peak vertical GRF changed significantly $(P=0.14$ and 0.77 , respectively). Thus we were confident that our apparatus applied external forces only in the horizontal direction. Furthermore, Davies (5) observed that, when subjects ran in a wind tunnel, they noticeably altered their posture by leaning into the wind. He noted that this leaning may have converted some potential drag forces into lift forces so that the external loads were no longer solely in the horizontal direction. Such a conversion of horizontal drag forces to vertical lift forces was not possible with our experimental apparatus.

Metabolic measurements. An open-flow system was used to measure the rate of $\mathrm{VO}_{2}$. Air was drawn from a loose-fitting mask to a variable-flow vacuum adjusted to draw air at a constant rate of $19.3 \mathrm{l} / \mathrm{s}$. We determined that this was an adequate flow rate because an increase in the main flow of the system did not change the measured $\mathrm{VO}_{2}$. The expired air was sampled continuously and analyzed for oxygen content by using an electronic oxygen analyzer (Ametek S3A-II). The system was calibrated before each experiment by bleeding nitrogen into the mask at a known rate $(0.2 \mathrm{l} / \mathrm{s})$, and the rate of $\mathrm{VO}_{2}$ was calculated according to the nitrogen dilution technique (8). $\mathrm{VO}_{2}$ values were corrected to standard temperature and pressure. All data were recorded with a microcomputer by using virtual instrumentation software (LabView 
4.0, National Instruments). Rates of $\dot{\mathrm{V}} \mathrm{O}_{2}$ were sampled continuously and averaged every $10 \mathrm{~s}$ during trials, Each trial lasted 8 min with adequate rest between trials. $\mathrm{VO}_{2}$ data for each trial were averaged only after steady state had been reached. We used an energetic equivalent of $20.1 \mathrm{~J} / \mathrm{ml}$ of oxygen (3).

Forcetreadmill measurements. This device consisted of a treadmill that was rigidly attached to a large force platform and enabled us to measure vertical and horizontal GRFs with high resolution. For each trial, we collected vertical and horizontal components of the GRF for $5 \mathrm{~s}$ at a rate of 1 $\mathrm{kHz} /$ channel. The GRF data were filtered by using a fourthorder recursive, zero phase-shift, Butterworth low-pass filter with a cutoff frequency of $25 \mathrm{~Hz}$. We previously determined that $99 \%$ of the integrated power content of the vertical GRF signal for normal running is at frequencies $<10 \mathrm{~Hz}$, whereas $98 \%$ of the horizontal GRF signal is at frequencies $<17 \mathrm{~Hz}$ (11). Filtered GRF data were adjusted such that the mean values for each component of GRF during the aerial phases were equal to zero.

Calculation of horizontal impulses. Horizontal braking and propulsive impulses were calculated for each trial ( 9 trials per subject). The first and last trials (zeroAHF) were averaged for each subject. Horizontal propulsive impulse data were obtained by integrating all the positive values of the horizontal GRF over the time of ground contact for 10 complete, successive step cycles. Similarly, horizontal braking impulse data were obtained by integrating all the negative values of the horizontal GRF over the time of ground contact for 10 complete, successive step cycles. A step was defined as ipsolateral heel strike to the next contralateral heel strike (i.e., one-half of a stride). These impulses were then each used to calculate an average horizontal braking and propulsive impulse per step.

In performing a study on the metabolic cost of generating horizontal forces, we recognize that there are many similarities between running on a level surface with an AHF and running on a hill. Despite some qualitative similarities between the two, there are some important quantitative differences between running on a hill and running on a level surface with an impeding or aiding force. An explanation of these quantitative differences is provided in the APPENDIX.

Statistical analysis. Metabolic and biomechanical data from this study were analyzed across all conditions of AHF by using a repeated measures design (ANOVA). We performed Tukey's honestly significant difference post hoc test to analyze the differences between each level of AHF. Significance was defined as $\mathrm{P}<0.05$.

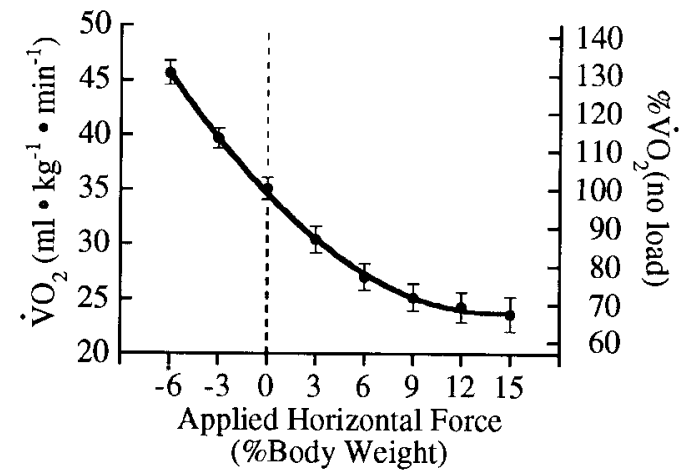

Fig. 2. Absolute rates of $\mathrm{O}_{2}$ consumption $\left(\dot{\mathrm{V}}_{2}\right.$, in $\left.\mathrm{ml} \cdot \mathrm{kg}^{-1} \cdot \mathrm{min}^{-1}\right)$ and relative rates of $\mathrm{V}_{2}(0 \%$ body wt AHF condition) vs. AHF (in $\%$ body wt). At the extreme impeding force ( $-6 \%$ body wt AHF), rate of $\mathrm{VO}_{2}$ increased to $130 \%$ of unloaded, control value. At the extreme aiding force ( $+15 \%$ body wt $\mathrm{AHF}$ ), rate of $\mathrm{V}_{2}$ decreased to $68 \%$ of unloaded, control value. Values indicate mean of 8 subjects for each condition. Error bars are SE of mean in absolute values $\left(\mathrm{ml} \cdot \mathrm{kg}^{-1} \cdot \mathrm{min}^{-1}\right)$. Curve represents a 2 nd-order least squares regression of all data points $\left(R^{2}=0.99\right)$.

\section{RESULTS}

Metabolic cost. For the zero AHF trials, subjects had an average $\mathrm{VO}_{2}$ of $35.1 \pm 1.0(\mathrm{SE}) \mathrm{ml} \cdot \mathrm{kg}^{-1} \cdot \mathrm{min}^{-1}$. The level of AHF exerted an overall significant effect on $\mathrm{VO}_{2}$ $(\mathrm{P}<0.0001)$, with $\mathrm{VO}_{2}$ increasing with greater impeding forces (-AHF; Fig. 2). A - 6\% BWt AHF resulted in a $30.2 \%$ increase in average metabolic rate compared with the zero AHF condition. I n contrast, $\mathrm{VO}_{2}$ decreased with increased aiding forces (+AHF; Fig. 2). A $+6 \%$ BWt AHF resulted in a $22.8 \%$ decrease in $\mathrm{VO}_{2}$, whereas a $+15 \%$ BWt AHF resulted in a $32.5 \%$ decrease in average metabolic cost over the zeroAHF trial. A Tukey honestly significant difference follow-up test revealed that the $\mathrm{VO}_{2}$ values at all but the $-3 \% \mathrm{BWt} A \mathrm{AHF}$ condition were significantly different from the control condition and that the $+6,+9,+12$, and $+15 \%$ BWt AHF were not significantly different from one another. A summary of these metabolic data appears in Table 1.

Biomechanics. Typical GRFs for unloaded running (zero AHF) and the extremes of impeding (-6\% BWt $\mathrm{AHF})$ and aiding (+15\% BWt AHF) conditions are

Table 1. Metabolic and kinetic data for different applied horizontal-force conditions

\begin{tabular}{|c|c|c|c|c|c|c|}
\hline $\begin{array}{c}\text { AHF } \\
\text { (\%body wt) }\end{array}$ & $\begin{array}{c}\mathrm{Vo}_{2} \\
\mathrm{ml} \cdot \mathrm{kg}^{-1} \cdot \mathrm{min}^{-1}\end{array}$ & $\begin{array}{c}\text { Braking } \\
\text { Impulse, } \\
\text { N/s }\end{array}$ & $\begin{array}{l}\text { Propulsive } \\
\text { Impulse, } \\
\text { N/s }\end{array}$ & $\begin{array}{l}\text { Peak } \\
\text { Braking } \\
\text { Force, } \\
\text { N }\end{array}$ & $\begin{array}{c}\text { Peak } \\
\text { Propulsive } \\
\text { Force, } \\
\text { N }\end{array}$ & $\begin{array}{l}\text { Peak } \\
\text { Vertical } \\
\text { Force, } \\
\text { N }\end{array}$ \\
\hline-3 & $39.7 \pm 0.9$ & $10.1 \pm 0.7$ & $16.9 \pm 1.1$ & $189.3 \pm 8.3$ & $210.9 \pm 10.9$ & $1,583 \pm 94$ \\
\hline 0 & $35.1 \pm 1.0$ & $13.9 \pm 0.6$ & $14.1 \pm 0.6$ & $227.2 \pm 6.6$ & $193.3 \pm 7.0$ & $1,670 \pm 72$ \\
\hline+3 & $30.4 \pm 1.2$ & $18.3 \pm 1.2$ & $11.7 \pm 0.9$ & $267.9 \pm 15.7$ & $172.3 \pm 10.5$ & $1,664 \pm 126$ \\
\hline+6 & $27.1 \pm 1.2$ & $22.9 \pm 1.7$ & $9.2 \pm 0.7$ & $293.8 \pm 15.0$ & $141.0 \pm 9.5$ & $1,633 \pm 128$ \\
\hline+15 & $23.7 \pm 1.6$ & $37.9 \pm 2.5$ & $4.2 \pm 0.6$ & $438.8 \pm 20.5$ & $83.3 \pm 8.1$ & $1,707 \pm 114$ \\
\hline
\end{tabular}

Average active peak of vertical ground reaction force (GRF) did not change significantly across all horizontal loading conditions $(P=0.77)$. In contrast, at an impeding force of $-6 \%$ body wt applied horizontal force (AHF), average peak braking GRF was $65 \%$ of zero AHF value and average peak propulsive GRF was $123 \%$ of control value. At an aiding force of $+15 \%$ body wt AHF, average peak braking GRF was $193 \%$ of control value and average peak propulsive GRF was $43 \%$ of control value. Values represent means for 8 subjects \pm SE of the mean. All variables showed significant trends $(\mathrm{P}<0.01)$ except for mean active peak of vertical GRF. 
shown in Fig. 3. The average horizontal impulses exerted per step for each condition are shown in Fig. 4. With an impeding force (-AHF), the horizontal braking impulse decreased, whereas the horizontal propulsive impulse increased. Conversely, the horizontal braking impulse increased, whereas the horizontal propulsive impulse decreased with aiding force (+AHF). As indicated in Fig. 3, the vertical GRF impact peak was altered appreciably with the level of AHF, but the active peak did not change. The impact (or passive) peak of the vertical GRF is thought to be caused by the collision of the heel (or the "effective mass" of the shank) with the ground (6). Despite these changes in the impact peak of the vertical GRF, the average vertical force per step for every trial was never $>1.6 \%$ different from the control condition. A summary of the kinetic data is provided in Table 1.

The typical horizontal GRF pattern for a person running at a steady speed on level ground consists of an initial braking phase (observed as a negative reaction force that decelerates the forward movement of the
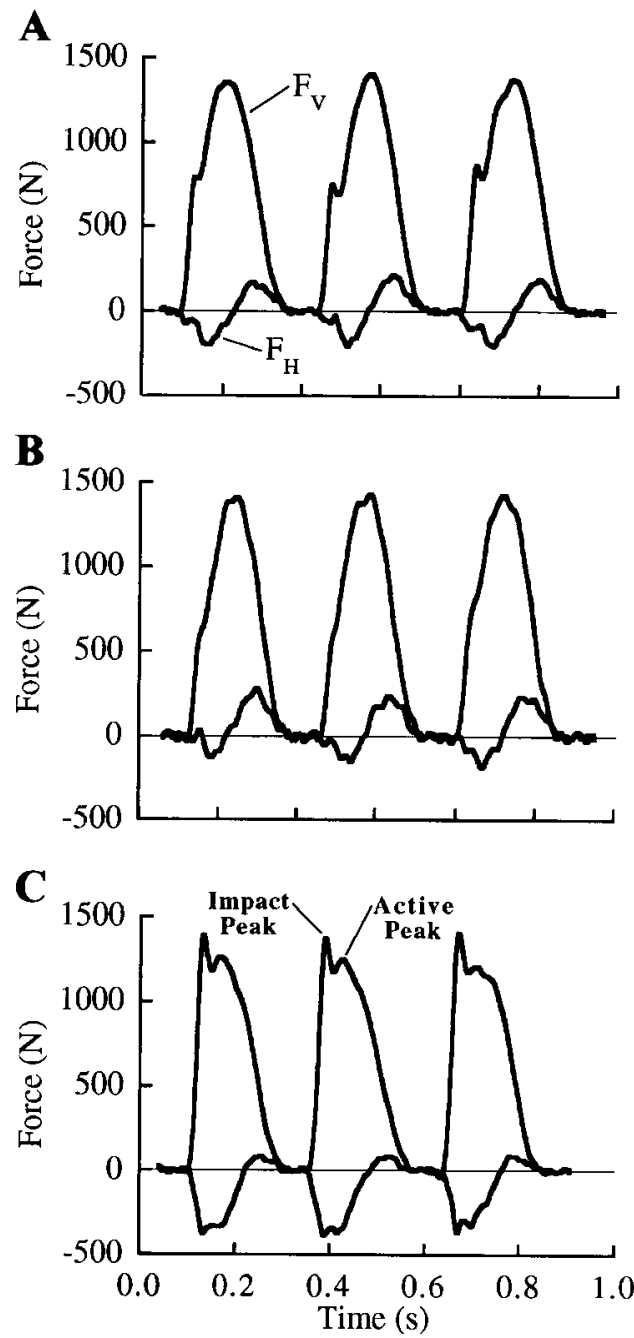

Fig. 3. Typical vertical $\left(F_{v}\right)$ and horizontal $\left(F_{h}\right)$ ground reaction forces for 3 steps of 1 subject: for unloaded, $0 \%$ body wt AHF control condition (A), with an impeding force of $6 \%$ body wt AHF (B), and with an aiding force of $15 \%$ body wt AHF (C).

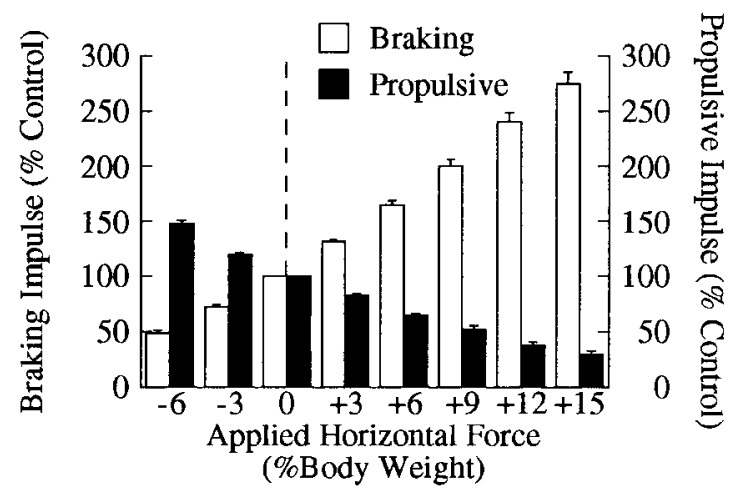

Fig. 4. Braking impulses and propulsive impulses vs. AHF (in \%body wt). All values are normalized to unl oaded, control condition ( $0 \%$ body wt AHF). Values are means for 8 subjects. Error bars represent SE of mean.

person) that is followed by a propulsive phase (observed as a positive reaction force that accelerates the person in the direction of travel; Ref. 17). N ote that, in the zero AHF case (Fig. 3A), the horizontal braking and propulsive impulses were equal and opposite as is necessary for steady-speed running on a level surface. Of course, this relationship did not hold when an AHF was used (Fig. 3, B and C), because the horizontal braking and propulsive impulses generated by the runner were altered to compensate for the external AHF to maintain a steady speed.

The stride kinematics of running were largely unaffected across all AHF conditions. The average stride frequency did not change significantly $(P=0.15)$ across all conditions. The average time of ground contact did change significantly $(P=0.03$ ) but never more than $\pm 0.01 \mathrm{~s}$ compared with the control condition. A summary of the kinematic data is provided in Table 2 .

\section{DISCUSSION}

At the greatest level of horizontal aiding force $(+15 \%$ BWt AHF), the metabolic rate of our subjects decreased by $33 \%$ from the metabolic rate of normal running. At this $+15 \%$ BWt AHF condition, the runners were

Table 2. Kinematic data for different applied horizontal-forceconditions

\begin{tabular}{cccc}
\hline \hline $\begin{array}{c}\text { AHF, } \\
\text { \% body wt }\end{array}$ & $\begin{array}{c}\text { Stride } \\
\text { Frequency, } \\
\mathrm{Hz}\end{array}$ & $\begin{array}{c}\text { Time of Contact } \\
\text { per Step, } \\
\text { s }\end{array}$ & Duty Factor \\
\hline-6 & $1.46 \pm 0.03$ & $0.268 \pm 0.009$ & $0.39 \pm 0.01$ \\
-3 & $1.45 \pm 0.03$ & $0.269 \pm 0.007$ & $0.39 \pm 0.01$ \\
0 & $1.40 \pm 0.02$ & $0.263 \pm 0.003$ & $0.37 \pm 0.01$ \\
+3 & $1.41 \pm 0.03$ & $0.258 \pm 0.006$ & $0.37 \pm 0.01$ \\
+6 & $1.41 \pm 0.03$ & $0.260 \pm 0.005$ & $0.37 \pm 0.01$ \\
+9 & $1.39 \pm 0.03$ & $0.261 \pm 0.007$ & $0.36 \pm 0.01$ \\
+12 & $1.40 \pm 0.03$ & $0.253 \pm 0.006$ & $0.35 \pm 0.01$ \\
+15 & $1.43 \pm 0.02$ & $0.253 \pm 0.006$ & $0.36 \pm 0.01$ \\
\hline
\end{tabular}

There were no substantial changes in kinematic variables measured at each horizontal loading condition. Average stride frequency did not change significantly $(P=0.15)$ across all conditions. Both average time of contact and average duty factor (fraction of stride period that 1 foot spends in contact with ground) showed slight but significant trends $(P=0.03)$. Values represent means for 8 subjects \pm SE of the mean. 
applying 70\% less propulsive impulse per step compared with what they applied without any AHF. This suggests that the metabolic cost of generating horizontal propulsive forces during normal running constitutes more than one-third of the total cost of steady-speed running. For this reason, we reject the hypothesis that the generation of horizontal forces during normal running has negl igible cost.

This decrease in metabolic rate with horizontal aiding forces occurred despite a dramatic increase in braking forces. For the $+15 \%$ BWt AHF condition, braking impulse increased by $172.7 \%$, yet we measured a net decrease in metabolic rate. Furthermore, with a horizontal impeding force of $-6 \% \mathrm{BWt}$, the metabolic rate increased by $30.2 \%$, despite a $51.1 \%$ decrease in braking impulse. At this condition, propulsive impulse increased $47.5 \%$. Therefore, it is plain to see that the generation of horizontal propulsive forces is metabolically much more expensive per unit of force than the generation of horizontal braking forces during steadyspeed running.

Generating horizontal forces was more expensive per unit of force than was generating vertical forces. For a $+15 \%$ BWt AHF, the average decrease in propulsive force generated per step was $57.5 \mathrm{~N}$. Expressed per unit of body mass, thecorresponding decreasein $\mathrm{VO}_{2}$ was $11.4 \mathrm{ml} \cdot \mathrm{kg}^{-1} \cdot \mathrm{min}^{-1}$. This decrease in the rate of $\mathrm{VO}_{2}$ is equivalent to $262.0 \mathrm{~W}$. Thus this suggests that generating $1 \mathrm{~N}$ of horizontal propulsive force on the ground during steady-speed running at $3.3 \mathrm{~m} / \mathrm{s}$ normally costs at least 4.6 W. Farley and McMahon (7) saw that, for a $25 \%$ decrease in average vertical force, there was a concomitant $25 \%$ decrease in $\mathrm{VO}_{2}$. Assuming a similar relationship for our data, we see that generating $1 \mathrm{~N}$ of vertical force on the ground during steady speed at $3.3 \mathrm{~m} / \mathrm{s}$ would cost $1.2 \mathrm{~W}$. Thus per unit of force generated, horizontal propulsive forces are al most four times more expensive than vertical forces. These results probably reflect the relative lengths of the moment arms over which these forces are applied. When moments (or torques) about each leg joint are considered, a unit of force generated horizontally on the ground would have a much greater effect than a unit of force generated vertically (especially at the more proximal joints).

At first glance, our findings seem to disagree with the data of Farley and McMahon (7) and Taylor and colleagues (22), who suggested a one-to-one relationship between metabolic cost and the generation of vertical forces. Their interpretation left little room for horizontal forces to play a role in determining the metabolic cost of running. Perhaps it is a naive notion to simply consider vertical and horizontal forces as separate determinants of the metabolic cost of running. It is the net resultant force generated on the ground that affects the net muscle moment at each joint as well as the force of each muscle crossing the joint. In reconciling this difference, we suggest that it may not be appropriate to consider vertical and horizontal GRFs as independent determinants of metabolic cost as they are conceptual rather than physiological constructs.
Future research investigating the influence of external perturbations on the metabolic cost of running should consider the effects of the resultant vector of the vertical and horizontal forces applied to the ground during level running.

\section{APPENDIX}

Qualitatively, both running against a horizontal impeding force and running uphill require more external work and, therefore, more power output by the runner. Similarly, a horizontal aiding force and downhill running require less external work and less power output by the runner. Both running with an AHF and running on a hill result in similar qualitative changes in metabolic cost. Nevertheless, there are critical differences between the two running conditions that reveal that they are not quantitatively the same.

One difference is in the efficiencies for each running condition. When running up a hill of some angle $(\Phi)$, gravity exerts an impeding force equal to $\mathrm{mg} \sin (\Phi)$, where $\mathrm{mg}=$ BWt. It is important to note that this calculation of the impeding force is performed with respect to the reference frame of the hill such that the angle of the hill defines the horizontal plane. With this equation, an "equivalent" hill angle $(\Phi)$ can be found where an impeding force equivalent to theAHF from our study can be cal culated. I n this way, we can calculate the work efficiencies as defined by Gaesser and Brooks (9) and compare them with similar values found in the literature for running both with impeding forces and along equivalent hill angles. Work efficiency is calculated as the external work rate divided by the corresponding increase in metabolic rate above the metabolic rate at zeroload (i.e., zero load = normal level running).

Our measurements of work efficiency are substantially higher than what has been reported for uphill running. At the $-3 \%$ BWt AHF, our data indicated a work efficiency of $62.6 \%$. Pugh (19) measured a similar work efficiency of $69.0 \%$ for running into a head wind at a similar mechanical power output, whereas he measured a work efficiency of $45.6 \%$ for running up an equivalent hill angle(i.e., at thesamemechanical power output). At the $-6 \% \mathrm{BWt}$ AHF, our data showed a work efficiency of $54.5 \%$. We calculated a substantially lower work efficiency of $46.6 \%$ for running up a hill angle equivalent to our $-6 \%$ BWt AHF by using the relationships reported by Bassett and colleagues (1). These data suggest that running on level ground with an AHF is quantitatively different from running up a hill

Some studies have suggested that the efficiencies for running with a horizontal force were the same as the efficiencies for uphill running $(5,13,23)$. These studies were difficult to compare and evaluate, however, because efficiency values were either averaged for a wide range of power outputs or else $\Delta$ efficiencies were given for comparison rather than work efficiencies ( $\Delta$ efficiency is defined as the change in work rate divided by the change in metabolic cost; Ref. 9).

A second difference between running with an AHF and uphill running is that the stride frequency does not change with the amount of AHF, whereas stride frequency increases considerably with increasing hill angles $(14,15)$. This suggests that the internal work (to swing the legs relative to the center of mass) for running with an AHF does not change with horizontal force, whereas internal work does increase with uphill running (14). This increase in internal work with hill angle may be one reason that work efficiency is lower for uphill running compared with running with an AHF.

A third important difference between running with an AHF and running up a hill is the posture that the runner adopts. In 
both cases, runners tend to align their trunks relative to the gravitational vector rather than relative to the ground. The runners in our study maintained an upright posture when running with a horizontal force, whereas I versen and McMahon (10) quantified how runners leaned forward relative to the reference frame of the hill angle.

Differences in posture probably change the orientation of the GRF vector relative to the leg joints. This would alter the effective mechanical advantage and thus the operation of the muscles and tendons during running (2). I ndeed, the differences in the efficiencies and stride kinematics for running with an AHF vs. running up a hill indicate that the muscles and tendons function differently in each condition. Normal running is a bouncing gait and involves the storage of both gravitational potential energy and horizontal kinetic energy as elastic energy. Running on the level with an AHF does not affect the change in gravitational potential energy, whereas running up a hill clearly does. The springlike mechanics of normal running are crucial for conserving metabolic energy. Roberts and colleagues (20) showed that turkeys utilize less elastic energy storage when running uphill than when running on level ground. The high efficiencies we observed suggest that our subjects retained this springlike behavior and were storing more elastic energy compared with humans running up a hill. For these reasons, running up a hill is quantitatively different from running with an AHF.

We thank Sabrina S. Selim for invaluable assistance in collecting the metabolic data. We also thank T. J. Roberts and the members of the University of California at Berkeley Locomotion Laboratory for insightful comments and suggestions.

This work was supported by National Institute of Arthritis and Musculoskeletal and Skin Diseases Grant R29-AR-44688 (to R. Kram).

Address for reprint requests and other correspondence: Y.-H. Chang, Locomotion Laboratory, Dept. of I ntegrative Biology, Univ. of California, 3060 Valley Life Sciences BIdg., Berkeley, CA 94720-3140 (E-mail: younghui@uclink4.berkeley.edu).

Received 10J uly 1998; accepted in final form 29 December 1998.

\section{REFERENCES}

1. Bassett, D. R., M. D. Giese, F. J . Nagle, A. Ward, D. M. Raab, and B. Balke. Aerobic requirements of overground versus treadmill running. Med. Sci. Sports Exerc. 17: 477-481, 1985.

2. Biewener, A. A. Scaling body support in mammals: limb posture and muscle mechanics. Science 245: 45-48, 1989.

3. Blaxter, K. Energy Metabolism in Animals and Man. Cambridge, UK: Cambridge University Press, 1989.

4. Cooke, C. B., M. J. McDonagh, A. M. Nevill, and C. T. Davies. Effects of load on oxygen intake in trained boys and men during treadmill running. J . Appl. Physiol. 71: 1237-1244, 1991.
5. Davies, C. T. Effects of wind assistance and resistance on the forward motion of a runner. J . Appl. Physiol. 48: 702-709, 1980

6. Denoth, J . Load on the locomotor system and modeling. In: Biomechanics of Running Shoes, edited by B. Nigg. Champaign, IL: Human Kinetics, 1986, p. 63-116.

7. Farley, C. T., and T. A. McMahon. Energetics of walking and running: insights from simulated reduced-gravity experiments. J . Appl. Physiol. 73: 2709-2712, 1992.

8. Fedak, M. A., L. Rome, and H. J. Seeherman. One-step $\mathrm{N}_{2}$-dilution technique for calibrating open-circuit $\mathrm{V}_{\mathrm{O}_{2}}$ measuring systems. J . Appl. Physiol. 51: 772- 776, 1981.

9. Gaesser, G. A., and G. A. Brooks. Muscular efficiency during steady-rate exercise: effects of speed and work rate. J. Appl. Physiol. 38: 1132-1139, 1975.

10. Iversen, J . R., and T. A. McMahon. Running on an incline. J . Biomech. Eng. 114: 435-441, 1992.

11. Kram, R., T. M. Griffin, J . M. Donelan, and Y. H. Chang. A force-treadmill for measuring vertical and horizontal ground reaction forces. J . Appl. Physiol. 85: 764- 769, 1998.

12. Kram, R., and C. R. Taylor. Energetics of running: a new perspective. Nature 346: 265-267, 1990.

13. Lloyd, B. B., and R. M. Zacks. The mechanical efficiency of treadmill running against a horizontal impeding force. J . Physiol . (Lond.) 223: 355-363, 1972.

14. Minetti, A. E., L. P. Ardigo, and F. Saibene. Mechanical determinants of the minimum energy cost of gradient running in humans. J . Exp. Biol. 195: 211-225, 1994.

15. Minetti, A. E., L. P. Ardigo, and F. Saibene. The transition between walking and running in humans: metabolic and mechanical aspects at different gradients. Acta Physiol. Scand. 150: 315-323, 1994

16. Morgan, D. W., P. E. Martin, G. S. Krahenbuhl, and F. D. Baldini. Variability in running economy and mechanics among trained male runners. Med. Sci. Sports Exerc. 23: 378- 383, 1991.

17. Munro, C. F., D. I. Miller, and A. J. Fuglevand. Ground reaction forces in running: a reexamination. J. Biomech. 20: 147-155, 1987.

18. Poole, D. C., and R. S. Richardson. Determinants of oxygen uptake. Sports Med. 24: 308-320, 1997.

19. Pugh, L. G. The influence of wind resistance in running and walking and the mechanical efficiency of work against horizontal or vertical forces. J. Physiol. (Lond.) 213: 255-276, 1971.

20. Roberts, T. J ., R. L. Marsh, P. G. Weyand, and C. R. Taylor. Muscular force in running turkeys: the economy of minimizing work. Science 275: 1113-1115, 1997.

21. Schieb, D. A. Kinematic accommodation of novice treadmill runners. Res. Q. Exerc. Sport 57: 49- 57, 1986.

22. Taylor, C. R., N. C. Heglund, T. A. McMahon, and T. R. Looney. Energetic cost of generating muscular force during running: a comparison of small and large animals. J . Exp. Biol. 86: $9-18,1980$

23. Zacks, R. M. The mechanical efficiency of running and bicycling against a horizontal impeding force. Int. Z. Angew. Physiol. Einschl. Arbeitsphysiol. 31: 249-258, 1973. 